\title{
Integrative Modeling of Oxidative Stress and C1 Metabolism Reveals Upregulation of Formaldehyde and Downregulation of Glutathione
}

\author{
Mrudhuula Mohan, Santhiya Kothandaram, Vyshali Venugopal, Prabhakar Deonikar, \\ V. A. Shiva Ayyadurai* \\ Systems Biology Group, International Center for Integrative Systems, Cambridge, MA, USA \\ Email: vashiva@integrativesystems.org
}

Received 5 June 2015; accepted 22 June 2015; published 25 June 2015

Copyright (C) 2015 by authors and Scientific Research Publishing Inc. This work is licensed under the Creative Commons Attribution International License (CC BY). http://creativecommons.org/licenses/by/4.0/

\section{Abstract}

This research provides, to the authors' knowledge, the first integrative model of oxidative stress and C1 metabolism in plants. Increased oxidative stress can cause irreversible damage to photosynthetic components and is harmful to plants. Perturbations at the genetic level may increase oxidative stress and upregulate antioxidant systems in plants. One of the key mechanisms involved in oxidative stress regulation is the ascorbate-glutathione cycle which operates in chloroplasts as well as the mitochondria and is responsible for removal of reactive oxygen species (ROS) generated during photosynthetic operations and respiration. In this research, the complexity of molecular pathway systems of oxidative stress is modeled and then integrated with a previously developed in silico model of C1 metabolism system. This molecular systems integration provides two important results: 1) demonstration of the scalability of the CytoSolve ${ }^{\circledR}$ Collaboratory $^{\mathrm{TM}}$, a computational systems biology platform that allows for modular integration of molecular pathway models, by coupling the in silico model of oxidative stress with the in silico model of C1 metabolism, and 2) derivation of new insights on the effects of oxidative stress on C1 metabolism relative to formaldehyde (HCHO), a toxic molecule, and glutathione (GSH), an important indicator of oxidative homeostasis in living systems. Previous in silico modeling of C1 metabolism, without oxidative stress, observed complete removal of formaldehyde via formaldehyde detoxification pathway and no change in glutathione concentrations. The results from this research of integrative oxidative stress with $\mathrm{C1}$ metabolism, however, demonstrate significant upregulation of formaldehyde concentrations, with concomitant downregulation and depletion of glutathione. Sensitivity analysis indicates that kGSH-HCHO, the rate constant of GSH-HCHO binding, VSHMT, the rate of formation of sarcosine from glycine, and $\mathrm{kO}_{2}^{-}$, the rate of superoxide formation significantly affect for-

\footnotetext{
${ }^{*}$ Corresponding author.
} 
maldehyde homeostasis in the C1 metabolism. Future research may employ this integrative model to explore which conditions initiate oxidative stress and the resultant upregulation and downregulation of formaldehyde and glutathione.

\title{
Keywords
}

\author{
C1 Metabolism, Oxidative Stress, Formaldehyde, Glutathione, Formaldehyde Detoxification, \\ Methionine Biosynthesis, Methylation Cycle, Computational Systems Biology, CytoSolve, Systems \\ Integration, Molecular Pathways
}

\section{Introduction}

The chemical and physiological process of oxidative stress occurring in all living systems is a result of excessive production and accumulation of reactive oxygen species (ROS) such as superoxide $\left(\mathrm{O}_{2}^{-}\right)$, hydrogen peroxide $\left(\mathrm{H}_{2} \mathrm{O}_{2}\right)$, hydroxyl radicals $(\mathrm{OH} \cdot)$, etc. [1]. This research provides, to the authors' knowledge, the first integrative model of oxidative stress and $\mathrm{C} 1$ metabolism in plants.

Increased oxidative stress can cause irreversible damage to photosynthetic component and is harmful to plants [2]. Additionally, any perturbations at the genetic level may increase oxidative stress and upregulate antioxidant systems in plants [3] [4]. One of the key mechanisms involved in oxidative stress regulation is the ascorbateglutathione cycle which operates in chloroplasts as well as the mitochondria and is responsible for removal of ROS generated during photosynthetic operations and respiration, respectively [5].

Given the significance of oxidative stress in plant metabolism, this study aims to explore, through the use of the CytoSolve ${ }^{\circledR}$ Collaboratory ${ }^{\mathrm{TM}}[6]$, a proven computational systems biology platform for scalable integration of molecular pathway models, how oxidative stress may affect C1 metabolism. C1 metabolism is a critical metabolic process in plants and is essential to provide one-carbon units for methylation and other types of modifications, as well as for nucleic acid, amino acid, and other biomolecule syntheses. This study builds on the previous work in the identification of critical molecular pathway systems, from a systematic bioinformatics review of C1 metabolism [7] and extends the in silico modeling of C1 metabolism [8], to incorporate effects of oxidative stress on key biomolecules involved in C1 metabolism.

An earlier systematic review of literature [7] resulted in the identification of three critical molecular pathway systems of C1 metabolism: 1) methionine biosynthesis, 2) the methylation cycle, and 3) formaldehyde detoxification. The in silico model of C1 metabolism [8] was derived from this earlier systematic review to develop a predictive and integrative computational model of C1 metabolism, also using the CytoSolve Collaboratory [6]. CytoSolve enabled the conversion of the diagrammatic representations of the three molecular pathway systems, identified in C1 metabolism from the earlier systematic review, into three individual molecular pathway models, which were then coupled and integrated dynamically using CytoSolve to produce the first comprehensive and integrated computational model of C1 metabolism [8]. The C1 metabolism modeling, for example, predicted the temporal behavior of formaldehyde, formate, sarcosine, glutathione, as well as many other key biomolecules involved in C1 metabolism, which may be hard to measure experimentally [8].

In this research, the complexity of molecular pathway systems of oxidative stress is modeled and then integrated with the recently developed in silico model of $\mathrm{C} 1$ metabolism in order: 1) to demonstrate the extensibility and scalability of the CytoSolve Collaboratory by coupling the in silico model of oxidative stress with the in silico model of C1 metabolism, and 2) to explore the effects of oxidative stress on C1 metabolism relative to formaldehyde (HCHO) and glutathione (GSH), a powerful antioxidant and important indicator of oxidative homeostasis in living systems.

\subsection{Brief Review of in Silico Modeling of C1 Metabolism}

Recent efforts have resulted in a comprehensive computational model of C1 metabolism [8]. Simulation results using the in silico model of $\mathrm{C} 1$ metabolism [8] provided new insights and predictions of temporal changes to formaldehyde, sarcosine and glutathione. The integrative model of C1 metabolism predicted that under normal conditions, formaldehyde is evanescently produced and detoxified rapidly between $\sim 1.5$ and $\sim 2$ days [8]. Gluta- 
thione levels are minimally affected and maintain a steady state of 5,000,000 nM. Finally, sarcosine is fully consumed during $\mathrm{C} 1$ metabolism. Parameter sensitivity analysis of the $\mathrm{C} 1$ metabolism model revealed that variations in kGSH-HCHO, the binding rate constant of glutathione (GSH) and formaldehyde (HCHO), affect formaldehyde concentration. The sensitivity analysis demonstrated that even an order of magnitude variation in this parameter still resulted in complete formaldehyde detoxification.

\subsection{Molecular Systems Integration with C1 Metabolism Model}

The systems architecture of C1 metabolism [7] provides a basis for future molecular systems integration with other molecular systems, such as: THF biosynthesis, oxidative stress metabolism, catalase activity, shikimic acid metabolism, adenosine metabolism, glyphosate metabolism, formate biosynthesis, and serine biosynthesis, based on the current literature review, which may affect and be affected by C1 metabolism. The in silico model of C1 metabolism offers a scalable and transparent research platform not only to study C1 metabolism, but also to expand and explore how other such molecular systems may affect and be affected by C1 metabolism.

In this research, oxidative stress, one such important molecular system, is investigated, modeled and integrated within the C1 metabolism model to explore the scalability of such systems integration. In addition, such integration may provide new insights to understand molecular mechanisms and their effects on key biomolecules in C1 metabolism.

\section{Methodology}

In this effort, the CytoSolve Collaboratory [6] is used to develop an in silico computational model of oxidative stress. First, a systematic bioinformatics literature review is conducted. Literature collection from an informatics standpoint is executed to ensure high recall to acquire the initial set. Based on the research question posed, 24 search criteria were developed as listed in Supplementary Materials Appendix A. The PubMed and Google Scholar databases were searched using the search criteria. This resulted in executions of 24 parallel independent searches to produce the initial set. Search refinement was executed to ensure increased precision to find relevant set of papers. A precision search was performed, by constraining the initial set to oxidative stress in plants within Titles or Abstracts, to acquire this relevant set.

The relevant papers were reviewed by domain experts through CytoSolve to determine the study set papers, which form the relevant set containing molecular pathway information such as:

1) description of species and reactions of oxidative stress

2) cellular compartments containing species and reactions

3) relevant enzymes

4) flux through oxidative stress

5) perturbations of oxidative stress

6) molecular pathways in oxidative stress

In this detection process, priority was given to those articles which were the most recent and which contained information and/or studies on oxidative stress and maize or closely related grasses. The final result of this literature review was to discover the key pathways of interest for oxidative stress.

Second, the molecular pathways found from the literature review provided the basis to develop a systems architecture, for oxidative stress. Third, the architecture was employed with CytoSolve to convert and integrate the molecular pathways of oxidative stress to create an in silico model of Oxidative Stress. Fourth, after validation and testing of the oxidative stress model, the model was then integrated with previously developed C1 Metabolism Model [8]. This resulted in an integrative model of oxidative stress and C1 metabolism. This model of oxidative stress and $\mathrm{C} 1$ metabolism, coupled together, was simulated to observe the effects on regulatory biomolecules.

All simulations were executed for a simulation time period of 800,000 seconds ( $\sim 9$ days). These simulations provide the insights for conducting in silico modeling and testing of biological phenomena to support in vitro and in vivo research.

\section{Results}

There are eight sets of results, which emerge from the research herein. The first set of results is a systematic literature review of oxidative stress, in Section 3.1.1. The second set of results is the identification of three mole- 
cular pathways systems comprising oxidative stress emerging from this systematic review, in Section 3.1.2. The third set of results is a systems architecture map of oxidative stress, presented in Section 3.1.3. The fourth set of results is an integrated systems architecture of C1 metabolism and oxidative stress, in Section 3.2, which reveals the interfaces of the interactions between the oxidative stress system and formaldehyde detoxification and methionine biosynthesis systems, the two of the three critical molecular systems of C1 metabolism.

The fifth and sixth set of results are simulation outputs from the integration of oxidative stress system separately with methionine biosynthesis and formaldehyde detoxification, in Sections 3.3.1 and 3.3.2, respectively. The seventh set of results is the simulation output from the integration of oxidative stress with the entire model of C1 metabolism, in Section 3.4.

Finally, the eighth set of results is the parameter sensitivity analysis, in Section 3.5, which provides a detailed understanding of which parameters are most sensitive to variations in the integrative computation of oxidative stress and C1 metabolism.

\subsection{In Silico Model of Oxidative Stress}

A standalone in silico model of oxidative stress in plants is developed using the following steps by: 1) performing systematic literature review of oxidative stress in plants, 2) identifying the key molecular pathway systems and convert them into validated mathematical models, and 3) developing a systems architecture for oxidative stress. The results from each of the steps are detailed in the sub-sections below.

\subsubsection{Literature Review of Oxidative Stress}

A systematic bioinformatics literature review is conducted for identification of oxidative stress pathways in plants similar to the method used to identify the key molecular pathways of C1 metabolism. Based on the framing of the research question and the application of the search criteria through a parallel strategy, the literature collection of an initial set of 279 papers is identified from online databases such as PubMed and Google Scholar. The final results of the systematic review are summarized in Figure 1.

\subsubsection{Identification of Molecular Pathways of Oxidative Stress}

Based on the systematic literature review, three major molecular systems that are involved in the oxidative stress pathway in plants are identified: These included: 1) lipid peroxidation pathway [9], 2) ascorbate-glutathione pathway [10], and 3) ROS production pathway [11].

The first molecular pathway system of oxidative stress that is identified is lipid peroxidation pathway and is shown in Figure 2. Lipids are the most abundant molecules in the membranes and hence the most probable targets of ROS. Oxidation of lipids occurs at their fatty acids, especially polyunsaturated fatty acids (PUFA) [9]. The oxidation of lipids by ROS produces various oxidized lipids (LOOH) species [12].

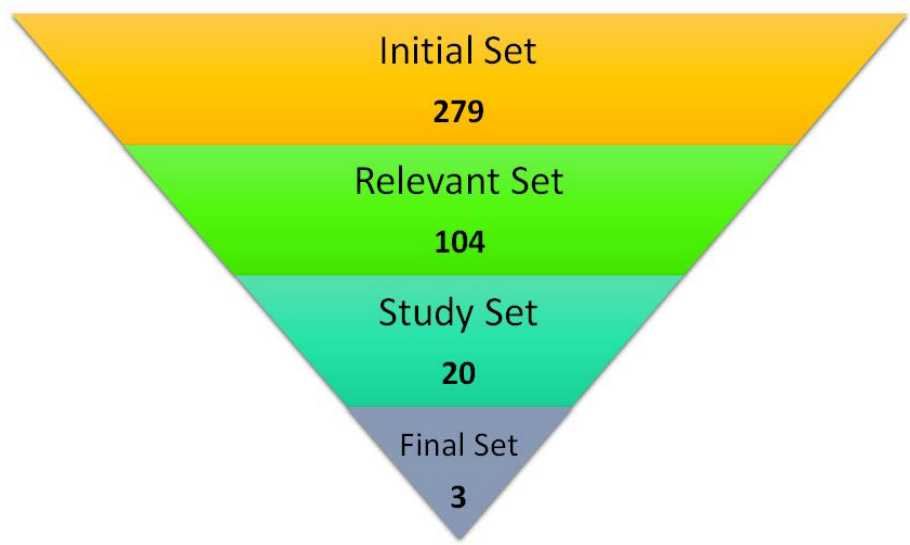

Figure 1. Systematic review results. There are 279 scientific papers (initial set), which met the search criteria. Of those, 104 papers (relevant set) appeared to be relevant based on the title and abstract. Upon further review, 20 papers (study set) were chosen as the study set upon which this systematic review is based. With this study set, 3 major molecular pathway systems (final set) from the study set were identified. 


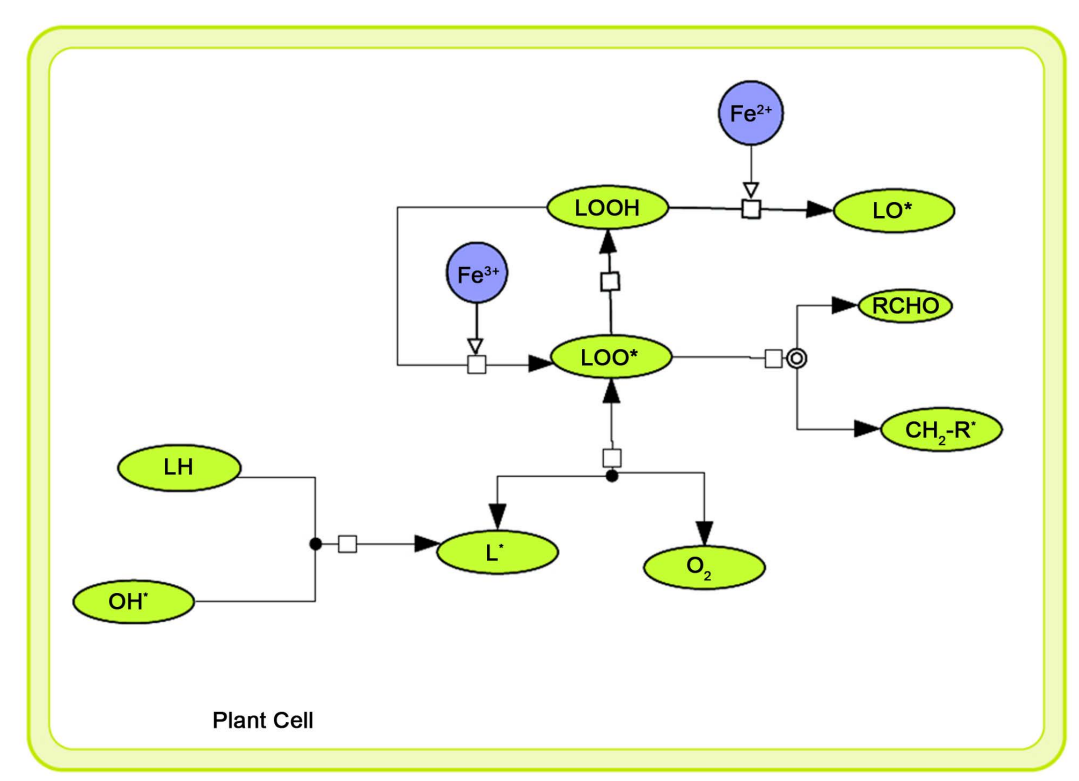

Figure 2. Lipid peroxidation pathway.

The second molecular pathway system of oxidative stress that is identified is ascorbate-glutathione cycle, as illustrated in Figure 3. It involves successive oxidations and reductions of ascorbate, glutathione, and NADPH by the enzymes ascorbate peroxidase, glutathione reductase, dehydroascorbate reductase, and monodehydroascorbate reductase. The removal of $\mathrm{H}_{2} \mathrm{O}_{2}$ produced in the chloroplast, as a result of the dismutation of $\mathrm{O}_{2}^{-} \cdot$, is essential to prevent inhibition of the Calvin cycle enzymes [3].

The third molecular pathway system that is identified is Reactive Oxygen Species (ROS) synthesis pathway, as shown in Figure 4. ROS in the form of $\mathrm{O}_{2}^{-}$radicals generates $\mathrm{H}_{2} \mathrm{O}_{2}$ by the action of the anti-oxidant enzyme superoxide dismutase (SOD) [11]. The enzyme catalase decomposes $\mathrm{H}_{2} \mathrm{O}_{2}$ into $\mathrm{H}_{2} \mathrm{O}$ and $\mathrm{O}_{2}$. However, in stress conditions, the Fenton reaction [11] is involved in the production of ROS in the form of highly reactive hydroxyl radicals $(\mathrm{OH} \cdot)$ from $\mathrm{H}_{2} \mathrm{O}_{2}$ with the simultaneous oxidation of iron from $\mathrm{Fe}^{2+}$ to the $\mathrm{Fe}^{3+}$ state. Radicals in the form of $\mathrm{HO}_{2}$. are also produced with the reduction of $\mathrm{Fe}^{2+}$ to the $\mathrm{Fe}^{3+}$. The $\mathrm{HO}_{2} \cdot$ can in turn give rise to $\mathrm{OH}$. and $\mathrm{H}_{2} \mathrm{O}_{2}$ in independent reactions [11].

\subsubsection{Systems Architecture of Molecular Systems of Oxidative Stress}

The systematic literature review for oxidative stress revealed that reactive oxygen species (ROS) synthesis, ascorbate-glutathione pathway and lipid peroxidation are integral part of oxidative stress pathways in plants. The systems architecture of oxidative stress, as shown in Figure 5, shows the interrelations between the molecular systems of oxidative stress pathways.

\subsection{Integrative Systems Architecture of Oxidative Stress and C1 Metabolism}

Earlier work on systematic review and modeling of C1 metabolism [7] [8] revealed the systems architecture for C1 metabolism. In Figure 6, an integrative molecular systems architecture is presented by coupling the systems architecture of oxidative stress with the systems architecture of C1 metabolism.

The coupled systems architecture reveals that oxidative stress and C1 metabolism interface through the ascorbate-glutathione pathway, which interfaces with the methionine biosynthesis and formaldehyde detoxification pathways of $\mathrm{C} 1$ metabolism. These interfaces will be relevant in developing and testing the in silico modeling of oxidative stress in C1 metabolism.

\subsection{Interaction of Oxidative Stress with Individual Molecular Pathways of C1 Metabolism}

Relative to the interface of oxidative stress with the methionine biosynthesis pathway, $\mathrm{H}_{2} \mathrm{O}_{2}$, a product of oxidative stress, is used to oxidizeglyoxylate, in the methionine biosynthesis pathway, to create formate [13]. 


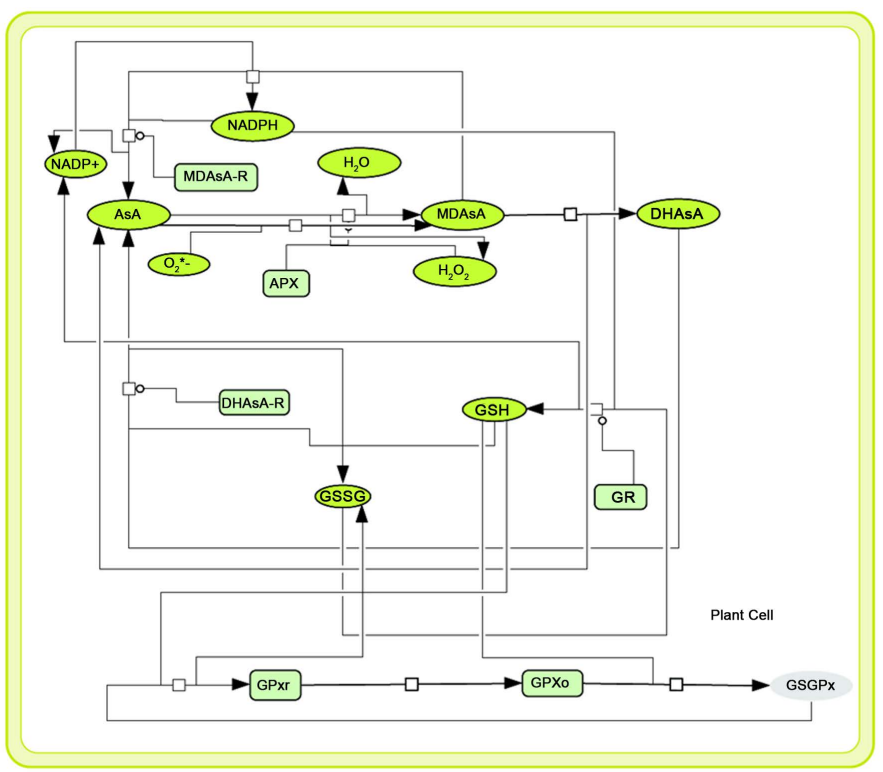

Figure 3. Ascorbate-glutathione pathway.

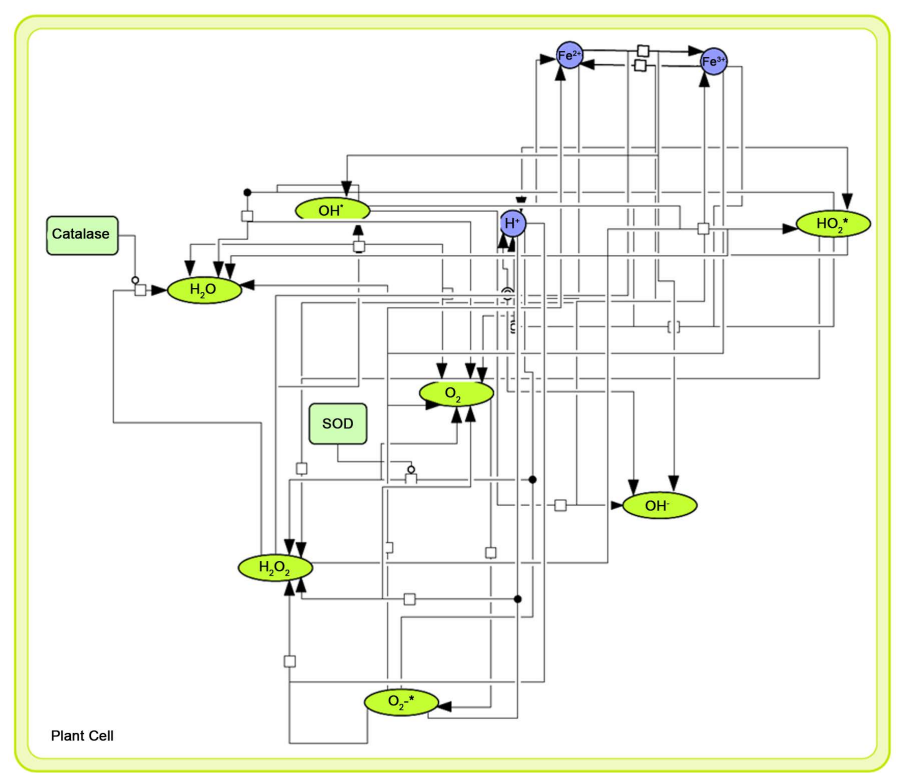

Figure 4. Reactive oxygen species (ROS) synthesis pathway.

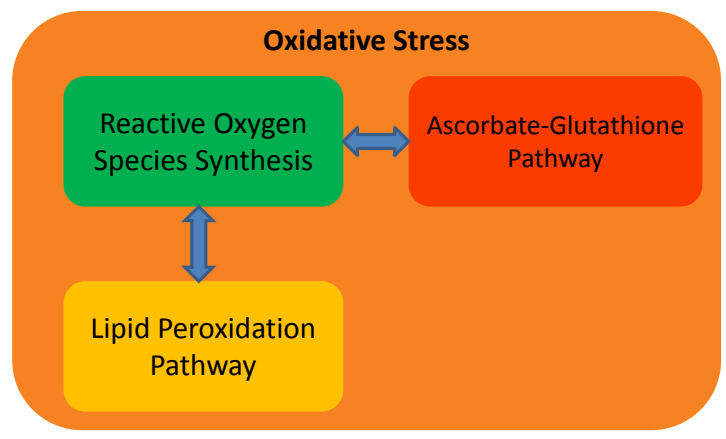

Figure 5. Systems architecture of oxidative stress. 


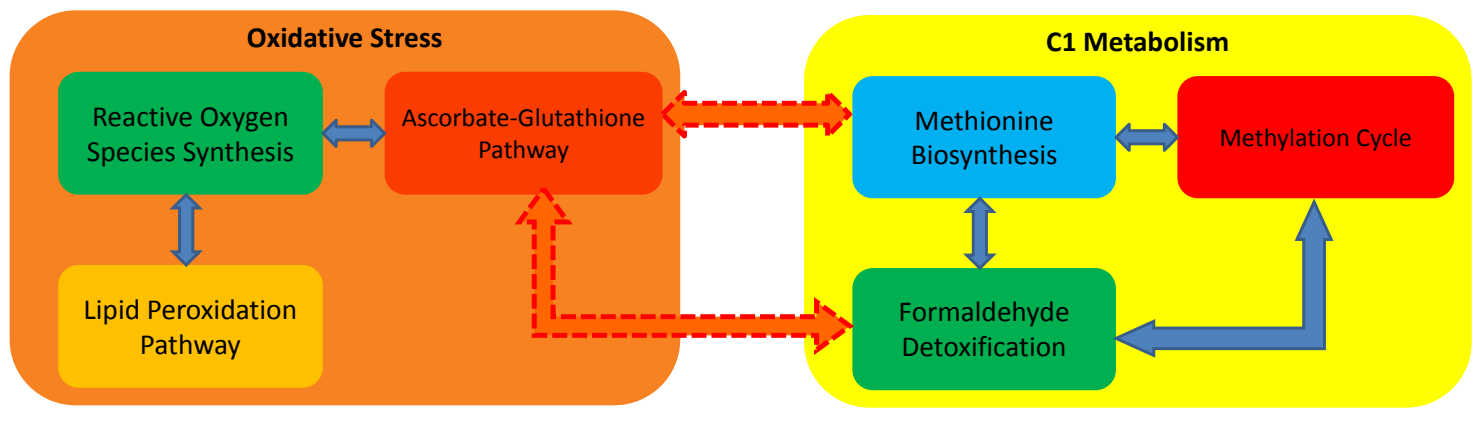

Figure 6. Systems architecture of oxidative stress pathway with oxidative stress.

Relative to the interface of oxidative stress with the formaldehyde detoxification pathway, glutathione, a main substrate for the antioxidant activity of glutathione reductase in the oxidative pathway [10] [14], binds with formaldehyde, which is the first step in clearing of formaldehyde [15]. Additionally, catalase, an antioxidant enzyme from oxidative stress pathway, catalyzes the conversion of methanol to formaldehyde [16]. All the models were simulated for a simulation time period of 800,000 seconds ( $\sim 9$ days).

\subsubsection{Simulation Results of in Silico Modeling of Oxidative Stress with Methionine Biosynthesis Pathways of C1 Metabolism}

Herein, the temporal dynamics of formaldehyde from in silico modeling of oxidative stress and integration with the methionine biosynthesis pathway, one of the three critical elements of the C1 metabolism, is presented in Figure 7.

The concentrations of formaldehyde, with or without oxidative stress, increase rapidly and reach a steady state at a concentration level of $\sim 0.06 \mathrm{nM}$. This result is consistent since methionine biosynthesis is a source of formaldehyde, and the production of formaldehyde will be not affected by any of the byproducts of oxidative stress. For example, $\mathrm{H}_{2} \mathrm{O}_{2}$, which is a product of oxidative stress, does not affect on the formation of formaldehyde in the methionine biosynthesis model, though $\mathrm{H}_{2} \mathrm{O}_{2}$ does affect oxidation of glyoxylate to formate [13].

\subsubsection{Simulation Results of in Silico Modeling of Oxidative Stress with Only Formaldehyde Detoxification Pathways of C1 Metabolism}

Herein, the temporal dynamics of formaldehyde and glutathione (GSH), from in silico modeling of oxidative stress and integration of the formaldehyde detoxification pathway, one of the three critical elements of the C1 metabolism, is presented in Figure 8(a) and Figure 8(b), respectively. The results show that there is an increase in formaldehyde concentrations (Figure 8(a)) and decrease in glutathione (Figure 8(b)) in presence of oxidative stress.

In Figure 8(a), the simulation results from integrating oxidative stress with the formaldehyde detoxification pathway of C1 metabolism indicate the formaldehyde concentration increases after a simulation period of $\sim 650,000$ seconds ( $\sim .5$ days) and reaches a level of $\sim 0.02 \mathrm{nM}$ at 800,000 seconds ( $\sim 9$ days). This result can likely be explained by understanding the dynamics of interactions between oxidative stress and formaldehyde detoxification. In the presence of oxidative stress, the synthesis of formaldehyde is increased and formaldehyde detoxification is lowered leading to accumulation of formaldehyde; however, there is a temporal delay in which the increases in formaldehyde concentrations become apparent.

This temporal delay of increased formaldehyde concentrations is likely because of the time evolution of two synergistic phenomena. The first phenomenon results from the accelerated consumption and depletion of glutathione (GSH) which results in reduced detoxification of formaldehyde. This is because there is a competitive need for glutathione (GSH) to be used: 1) to clear $\mathrm{H}_{2} \mathrm{O}_{2}$, a byproduct of oxidative stress [10], and, 2) to enable the detoxification of formaldehyde [17]. The second phenomenon results from the increased production of formaldehyde from the increased conversion of methanol to formaldehyde by catalase, which is an important and integral enzyme of oxidative stress pathway [10] [14] [15]. The coupling of oxidative stress with formaldehyde detoxification exposes catalase, which was originally non-existent within the formaldehyde detoxification pathway alone. 


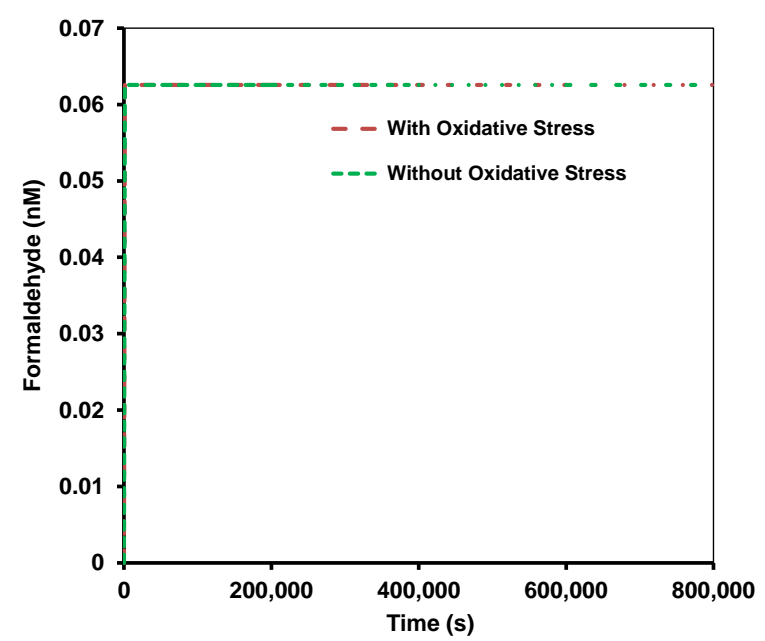

Figure 7. Simulation results of formaldehyde concentration in methionine biosynthesis model with and without oxidative stress.

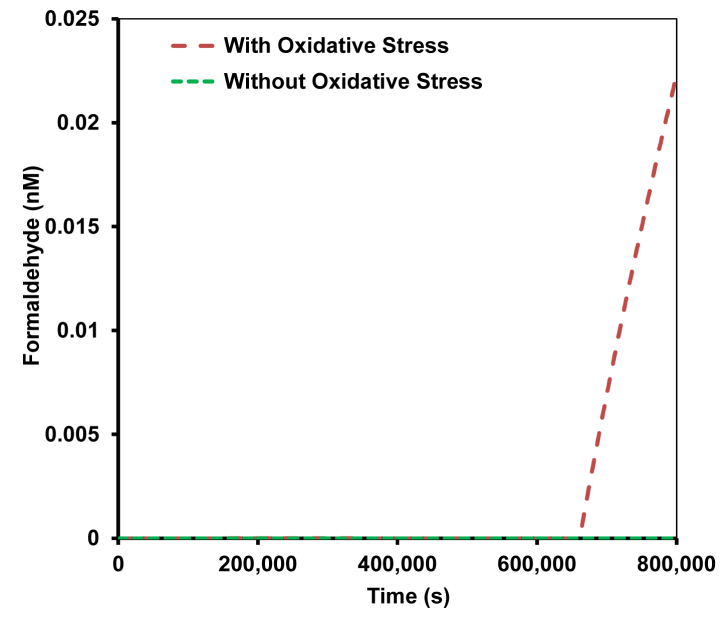

(a)

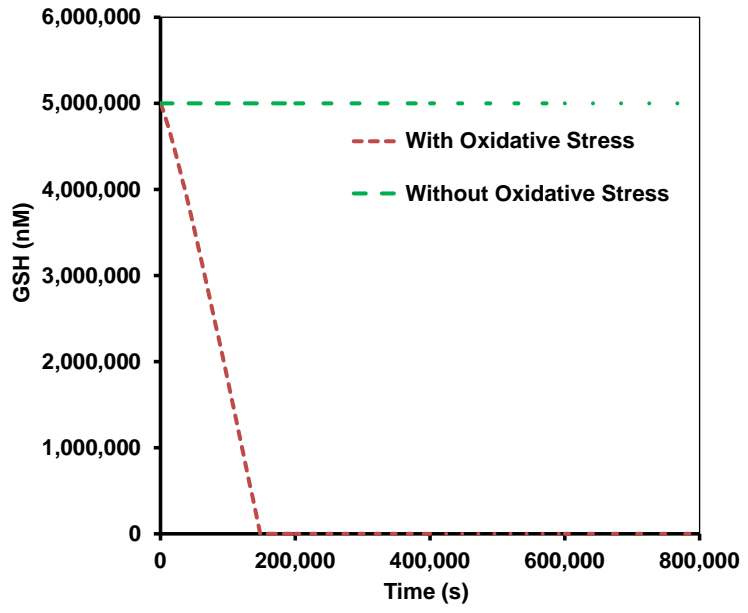

(b)

Figure 8. (a) Simulation results of formaldehyde concentration in formaldehyde detoxification model with and without oxidative stress; (b) Simulation results of glutathione (GSH) concentration in formaldehyde detoxification model with and without oxidative stress.

In summary, oxidative stress affects the formaldehyde detoxification pathway by increasing formaldehyde concentrations, synergistically through reducing formaldehyde clearance and increasing formaldehyde synthesis.

In Figure 8(b), the simulation results, from integrating oxidative stress with the formaldehyde detoxification pathway of $\mathrm{C} 1$ metabolism, indicate that glutathione (GSH) concentration varies significantly with and without the presence of oxidative stress. Without the presence of oxidative stress, glutathione levels remain at the steady state value of 5,000,000 $\mathrm{nM}$. With the presence of oxidative stress, glutathione is completely depleted within $\sim 180,000$ seconds ( 2 days).

This result can likely be explained by understanding the dynamics of glutathione's dual role in oxidative stress as well as formaldehyde detoxification. In this simulation, an initial and finite amount of glutathione is provided, which is not replenished. In the oxidative stress molecular system alone, where glutathione (GSH) is needed to clear $\mathrm{H}_{2} \mathrm{O}_{2}$ [10], a byproduct of oxidative stress, glutathione levels will decrease over time. In the formaldehyde detoxification molecular system, where glutathione is needed to clear and detoxify formaldehyde accumulation [14], glutathione is used and replenished in a cycle with a temporal periodicity. The simulation reveals that for a finite and initial amount of glutathione, the integration of oxidative stress with formaldehyde detoxification will eventually lead to depletion of glutathione, notwithstanding any new sources of glutathione 
production.

In summary, oxidative stress significantly perturbs the homeostasis of the glutathione in formaldehyde detoxification.

\subsection{Simulation Results of in Silico Modeling of Oxidative Stress with Only Integrative Model of C1 Metabolism}

The previous section 3.3 provided simulation results from the integration of oxidative stress independently with methionine biosynthesis and formaldehyde detoxification. In this section, we present the simulation results of the holistic integration of the molecular system of oxidative stress with the entire C1 metabolism system. The simulations are executed for a simulation time period of 800,000 seconds ( $\sim 9$ days).

The simulation results from the integration of oxidative stress with the complete C1 metabolism system reveal increases in formaldehyde accumulation and concomitant glutathione depletion, as shown in Figure 9(a) and Figure 9(b), respectively.

In Figure 9(a), the simulation of the molecular systems integration of oxidative stress and the C1 metabolism indicate that formaldehyde concentration varies significantly with and without the presence of oxidative stress. Without the presence of oxidative stress, formaldehyde does not accumulate in the C1 metabolism system. In the presence of oxidative stress, formaldehyde accumulates in the C1 metabolism system, starting at $\sim 180,000$ seconds ( $\sim 2$ days) and continues accumulating non-linearly to $\sim 13 \mathrm{n}$ Min 800,000 seconds ( $\sim 9$ day).

This simulation result is consistent with the previous integration of with and without oxidative stress in individual model of formaldehyde detoxification. There are two key differences, however, in the temporal accumulation of formaldehyde in the integrative model of oxidative stress and C1 metabolism (Figure 9(a)) versus the interaction of oxidative stress with formaldehyde detoxification alone (Figure 8(a)). The first difference is that in the integrative model of oxidative stress and C1 metabolism, formaldehyde accumulation begins nearly 5 times sooner at $\sim 180,000$ seconds ( $\sim 2$ days) versus at $\sim 650,000$ seconds ( $\sim 7.5$ days). The second difference is that at 800,000 seconds ( $\sim 9$ days), the formaldehyde concentration in the integrative model of oxidative stress and C1 metabolism is $\sim 700$ times higher at $\sim 13 \mathrm{nM}$ versus at $\sim 0.02 \mathrm{nM}$.

These results indicate that in the integrative model of oxidative stress and C1 metabolism, formaldehyde accumulation occurs faster and achieves a significantly higher level during the same temporal period. This is likely due to the fact that, in the $\mathrm{C} 1$ metabolism model, not only are the effects on the formaldehyde detoxification being considered, but also its coupled effects with methionine biosynthesis and the activated methyl cycle. The activated methyl cycle contributes to formaldehyde production from sarcosine [18]. In summary, oxidative stress affects C1 metabolism by not only increasing the rate and quantity of formaldehyde concentrations through the activated methyl cycle, for example, but also by reducing the rate and quantity of formaldehyde clearance in the formaldehyde detoxification pathway.

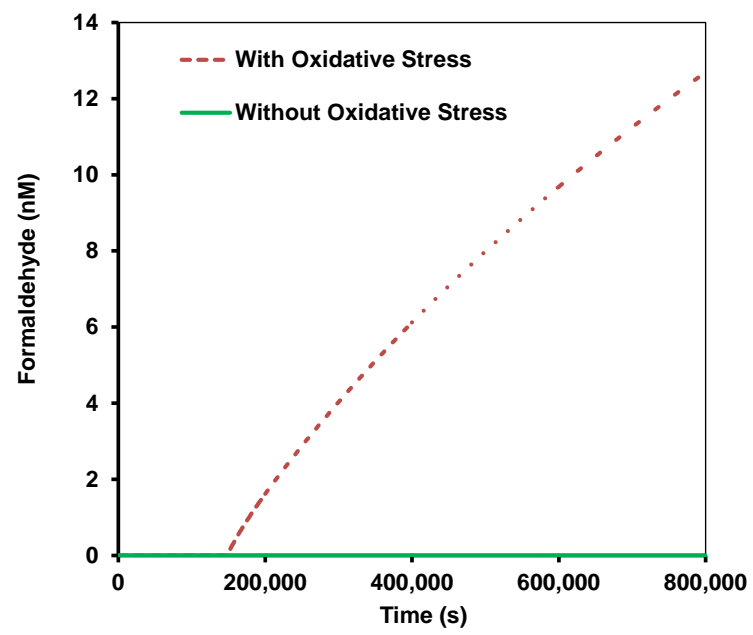

(a)

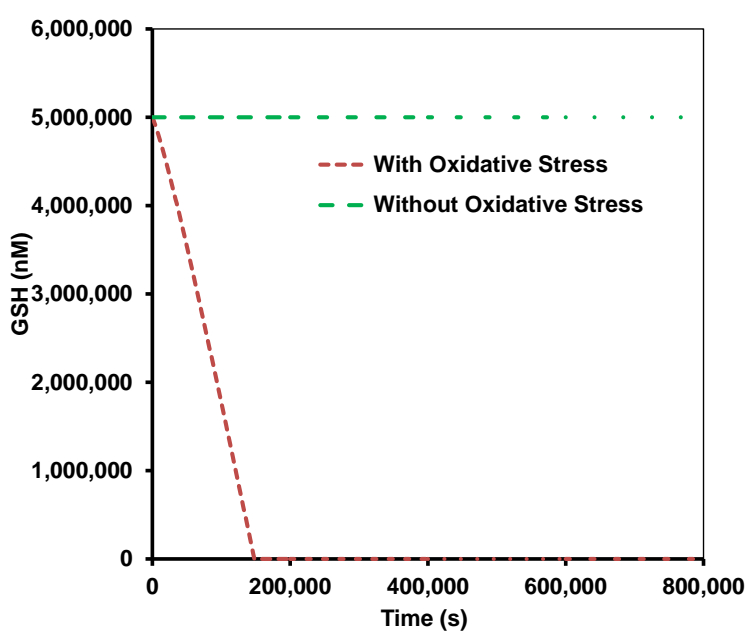

(b)

Figure 9. (a) Simulation results of formaldehyde concentration in C1 metabolism model with and without oxidative stress; (b) Simulation results of glutathione (GSH) concentration from integrative C1 metabolism model. 
In Figure 9(b), the simulation of the molecular systems integration of oxidative stress and C1 metabolism indicate that glutathione (GSH) concentrations vary significantly with and without the presence of oxidative stress. Without the presence of oxidative stress, glutathione levels remain at the steady state value of 5,000,000 nM. With the presence of oxidative stress, glutathione is completely depleted within $\sim 180,000$ seconds ( 2 days).

This simulation result is consistent with the previous integration of with and without oxidative stress in the individual model of formaldehyde detoxification. In this simulation, an initial and finite amount of glutathione is provided, which is not replenished. In the oxidative stress molecular system alone, where glutathione (GSH) is needed to clear $\mathrm{H}_{2} \mathrm{O}_{2}$, a byproduct of oxidative stress, glutathione levels will decrease over time. Simulation reveals that for a finite initial amount of glutathione, the integration of oxidative stress with C1 metabolism will eventually lead to depletion of glutathione, notwithstanding any new sources of glutathione production. The close similarity of this result (Figure 9(b)) with the previous result (Figure 8(b)) is because glutathione (GSH) directly affects and couples oxidative stress and formaldehyde detoxification, and is decoupled from methionine biosynthesis and the activated methyl cycle.

\subsection{Parameter Sensitivity of C1 Metabolism with Oxidative Stress Model}

The results from simulations of the molecular systems integration of oxidative stress and the C1 metabolism provide insights on two key biomolecular species: formaldehyde and glutathione. The integrity of literature reviewed and the kinetic rate constants used in the modeling is critical for the interpretation and usefulness of the simulation results. The relative significance of these critical parameters can be assessed by conducting a parameter sensitivity analysis.

Given the importance of formaldehyde synthesis and clearance in C1 metabolism, and central role of glutathione (GSH) in the oxidative stress homeostasis, the effect of four critical parameters was tested on formaldehyde and glutathione concentrations. These parameters are:

1. VCAT-Rate of formaldehyde production from methanol

2. kGSH-HCHO-Binding rate constant of glutathione (GSH) and formaldehyde (HCHO)

3. VGMT - Rate of production of sarcosine from glycine

4. $\mathrm{kO}_{2}^{-}$- Rate of production of superoxide

Four sets of results emerge from the parameter sensitivity analysis of the four parameters itemized above. The first parameter that is analyzed is VCAT. VCAT is varied from 22 to $100 \mathrm{nM} \cdot \mathrm{s}^{-1}$ and the resulting formaldehyde and glutathione (GSH) concentrations are observed for the integrated oxidative stress and C1 metabolism model in Figure 10(a) and Figure 10(b), respectively.

These results indicate that both formaldehyde and glutathione concentrations are not sensitive to changes in VCAT for the integrated oxidative stress and C1 metabolism model. In all cases, formaldehyde accumulates to the same levels, and glutathione (GSH) is fully depleted.

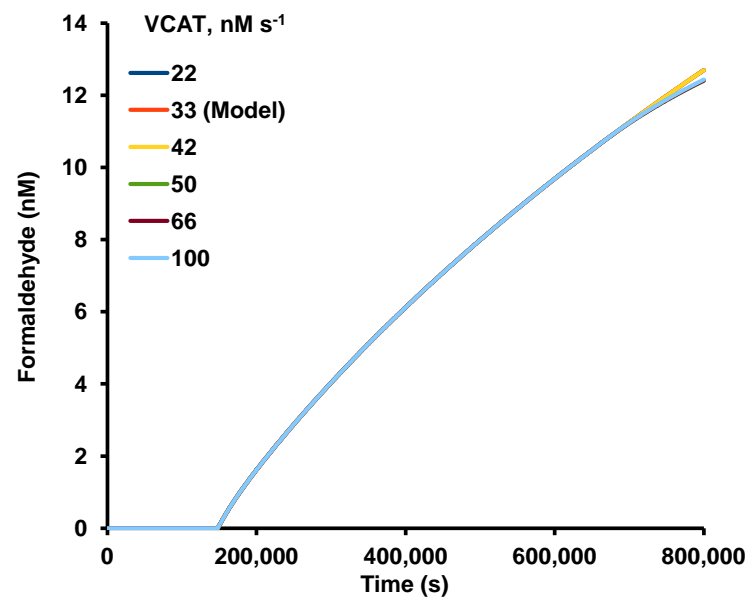

(a)

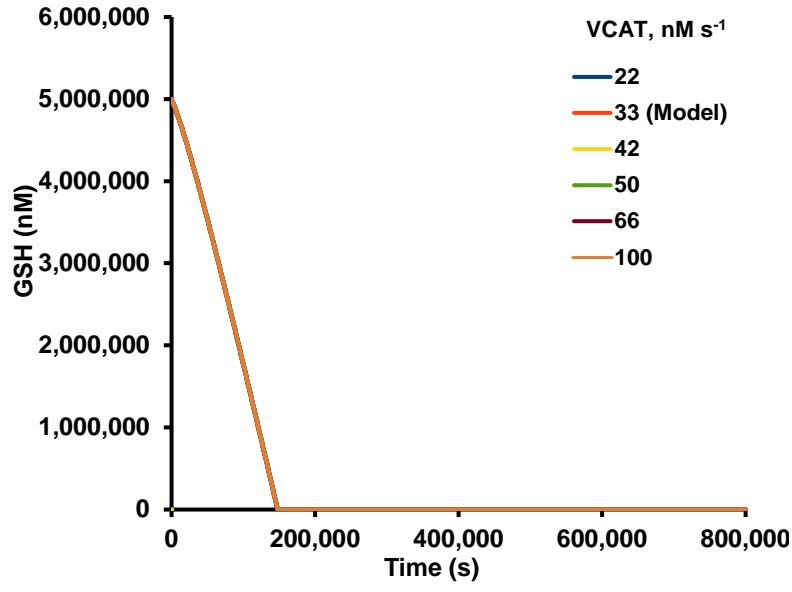

(b)

Figure 10. (a) Parameter sensitivity analysis of VCAT on formaldehyde in the integrated oxidative stress and C1 metabolism model; (b) Parameter sensitivity analysis of VCAT on glutathione (GSH) in the integrated oxidative stress and C1 metabolism model. 
The second parameter that is varied is kGSH-HCHO. kGSH-HCHO is varied from 0.000864 to 0.00864 $\mathrm{n} \cdot \mathrm{M}^{-1} \cdot \mathrm{s}^{-1}$ and the resulting formaldehyde and glutathione concentrations are observed for the integrated oxidative stress and C1 metabolism model in Figure 11(a) and Figure 11(b), respectively.

These results indicate that kGSH-HCHO is sensitive and affects formaldehyde concentrations. As Figure 11(a) illustrates, an order of magnitude variation kGSH-HCHO results in a non-linear variation at $~ 800,000$ seconds ( $\sim 9$ days) of formaldehyde concentrations by four times. These results, relative to glutathione (GSH), however, indicate that kGSH-HCHO is insensitive and does not affect glutathione (GSH) concentrations. In all cases, formaldehyde accumulates though to varying levels, and glutathione (GSH) is fully depleted.

The third parameter that is varied is VMTG. VMTG is varied from 20 to $87 \mathrm{nM} \cdot \mathrm{s}^{-1}$ and the resulting formaldehyde and glutathione concentrations are observed for the integrated oxidative stress and C1 metabolism model in Figure 12(a) and Figure 12(b), respectively.

These results indicate that VMTG is sensitive and affects formaldehyde concentrations. As Figure 12(a) illustrates, a four times variation of VMTG results in a nonlinear variation at $\sim 800,000$ seconds ( $\sim 9$ days) of formaldehyde concentrations by six times. In all cases, formaldehyde is shown to accumulate consistently, and is never depleted. These results, relative to glutathione (GSH), however, indicate that VMTG is insensitive and

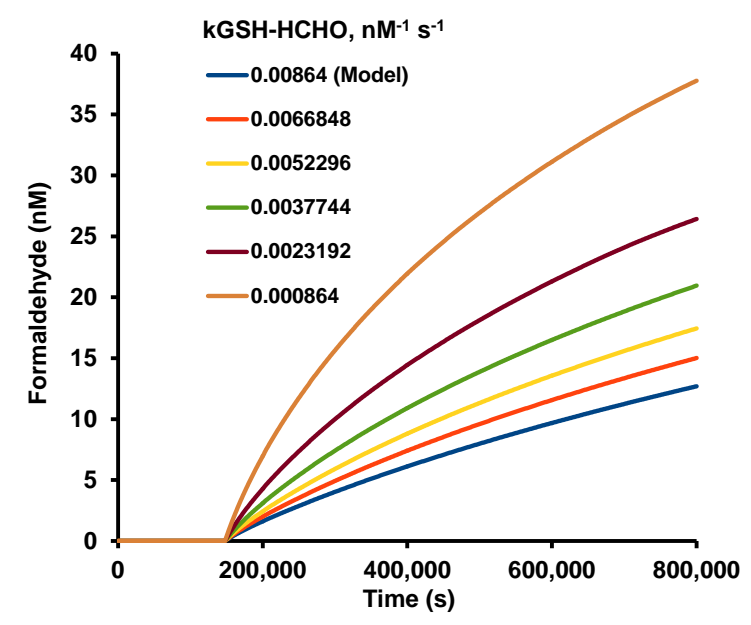

(a)

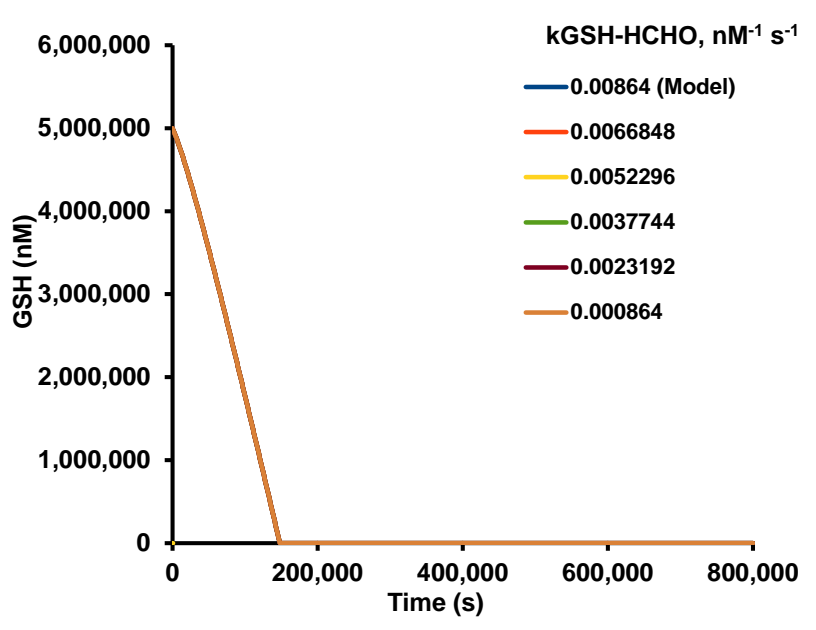

(b)

Figure 11. (a) Parameter sensitivity analysis of kGSH-HCHO on formaldehyde in the integrated oxidative stress and C1 metabolism model; (b) Parameter sensitivity analysis of kGSH-HCHO on glutathione (GSH) in the Integrated oxidative stress and C1 metabolism model.

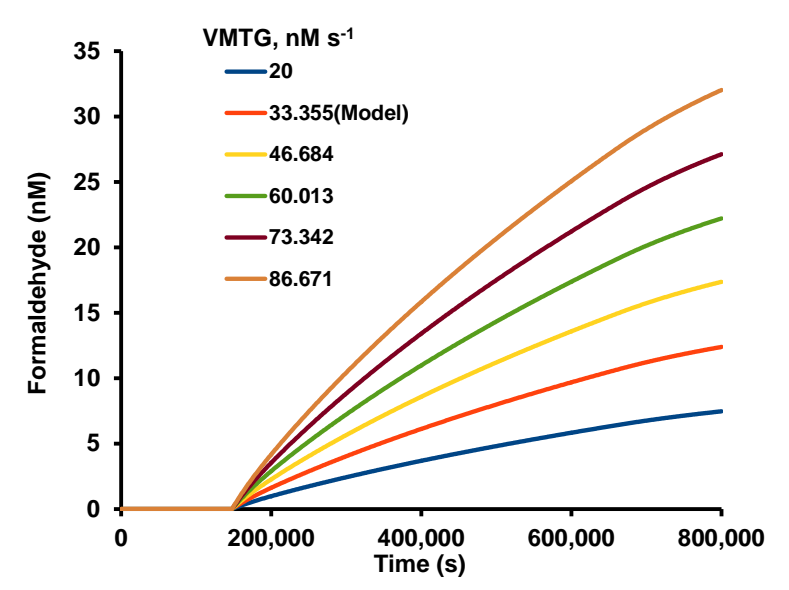

(a)

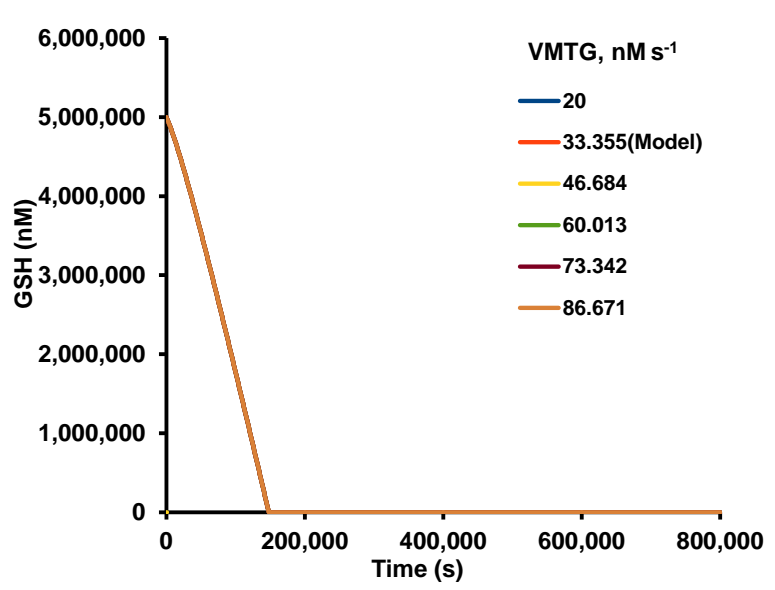

(b)

Figure 12. (a) Parameter sensitivity analysis of VMTG on formaldehyde in the integrated oxidative stress and C1 metabolism model; (b) Parameter sensitivity analysis of VMTG on glutathione (GSH) in the integrated oxidative stress and C1 metabolism model. 
does not affect glutathione (GSH) concentrations. In all cases, formaldehyde accumulates though to varying levels, and glutathione (GSH) is fully depleted.

The fourth parameter that is varied is $\mathrm{kO}_{2}^{-}$. $\mathrm{kO}_{2}^{-}$is varied from 20 to $100 \mathrm{nM} \cdot \mathrm{s}^{-1}$ and the resulting formaldehyde and glutathione concentrations are observed for the integrated oxidative stress and C1 metabolism model in in Figure 13(a) and Figure 13(b), respectively.

These results indicate that $\mathrm{kO}_{2}^{-}$is sensitive and affects formaldehyde concentrations. As Figure 13(a) illustrates, a five times variation of $\mathrm{kO}_{2}^{-}$results in a non-linear variation at $\sim 800,000$ seconds ( $\sim 9$ days) of formaldehyde concentrations by twenty times. These results indicate that $\mathrm{kO}_{2}^{-}$is sensitive and affects glutathione (GSH) concentrations. As Figure 13(b) illustrates, a five times variation of $\mathrm{kO}_{2}^{-}$results in an acceleration of glutathione depletion by a factor of seven. In all cases, formaldehyde accumulates though to varying levels, and glutathione (GSH) is fully depleted, though temporally accelerated to varying degrees.

\section{Discussion and Conclusions}

This research aimed to perform computational molecular systems integration of oxidative stress with the recently developed in silico model of C1 metabolism in order: 1) to demonstrate the extensibility and scalability of the in silico model of C1 metabolism resident in the CytoSolve Collaboratory, and 2) to explore the effects of oxidative stress on $\mathrm{C} 1$ metabolism relative to formaldehyde (HCHO) and glutathione (GSH), a powerful antioxidant and important indicator of oxidative homeostasis in living systems.

Relative to the first aim, the CytoSolve platform and process were used to perform a systematic literature review to derive three critical molecular pathway systems of oxidative stress: 1) lipid peroxidation pathway, 2) ascorbate-glutathione pathway, and 3) ROS production pathway. These three molecular systems were then integrated to produce an integrative computational model of oxidative stress. This effort served to define an integrative systems architecture that derived critical interfaces of the oxidative stress system with the molecular systems of formaldehyde detoxification and methionine biosynthesis, two of the three critical components of C1 metabolism.

Integration and simulation results from in silico modeling of oxidative stress with the individual molecular system of formaldehyde detoxification demonstrated that oxidative stress caused accumulation of formaldehyde concentrations, and depleted glutathione. Integration and simulation results from in silico modeling of oxidative stress with the individual molecular system of methionine biosynthesis demonstrated that oxidative stress neither affected the accumulation of formaldehyde nor depletion of glutathione in methionine biosynthesis.

Relative to the second aim, the computational molecular systems integration of oxidative stress with the fully integrative model of C1 metabolism revealed that oxidative stress significantly increased formaldehyde accumulation while concomitantly depleting glutathione. Moreover, parameter sensitivity analysis demonstrated that

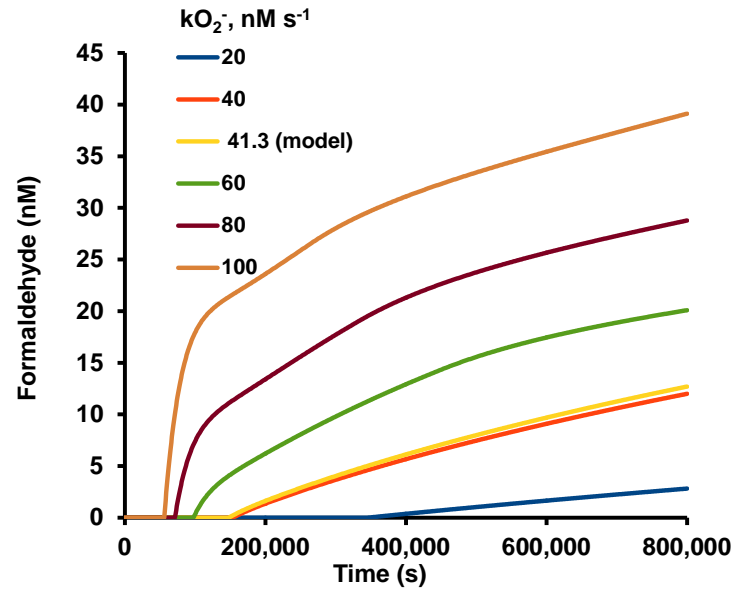

(a)

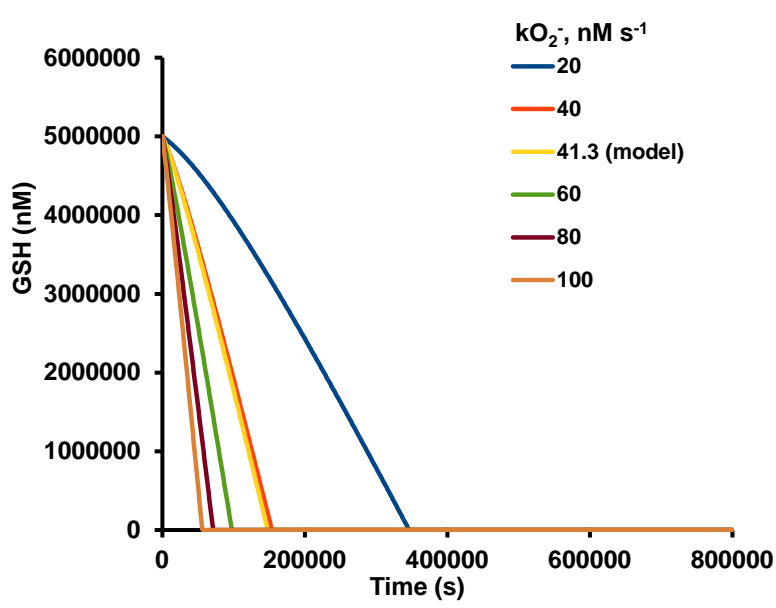

(b)

Figure 13. (a) Parameter sensitivity analysis of $\mathrm{kO}_{2}^{-}$on formaldehyde in the integrated oxidative stress and $\mathrm{C} 1$ metabolism model; (b) Parameter sensitivity analysis of $\mathrm{kO}_{2}^{-}$on glutathione (GSH) in the integrated oxidative stress and C1 metabolism model. 
formaldehyde accumulation, as well as glutathione depletion were observed consistently, even with variations to critical parameters such as VCAT, kGSH-HCHO, VMTG and $\mathrm{kO}_{2}^{-}$.

In summary, the molecular systems integration of oxidative stress and $\mathrm{C} 1$ metabolism provides two critical insights: 1) formaldehyde detoxification in $\mathrm{C} 1$ metabolism is perturbed by oxidative stress resulting in significant up gregulation of formaldehyde (Figure 9(a), Figure 10(a), Figure 11(a), Figure 12(a) and Figure 13(a)), and 2) gluthatione production is perturbed resulting in its significant downregulation and depletion (Figure 9(b), Figure 10(b), Figure 11(b), Figure 12(b) and Figure 13(b)). The results from this research are in stark contrast with the in silico analysis and simulation results of C1 metabolism, without oxidative stress [7], which observed complete detoxification and removal of formaldehyde and maintenance of high glutathione levels, without any depletion of glutathione.

\section{Future Directions}

This research demonstrates the modular extensibility and scalability of the CytoSolve Collaboratory for advancing scientific research in the field of plant biology using principles of computational molecular systems biology. The in silico modeling efforts, earlier performed in deriving and establishing the C1 metabolism model [7] [8], was itself an example of using systems engineering principles to interconnect, in a "plug and play" fashion, three complex molecular systems to derive a larger and more complex system.

Plant biology is complex with molecular phenomena spanning multiple spatial and temporal scales, across multiple domains of expertise. Given the nature of biological endeavor where one must focus on specificity and domain expertise to make contributions, the notion of "systems integration" and "collaboration" is relatively a novel concept in practice.

Research efforts, as the one herein, can perhaps provide motivation and guidance to the biological research community that in silico modeling, when performed in a modular and integrative manner, by employing a systematic bioinformatics literature review, can provide a mechanism for: 1) generalized research and study of complex biological phenomena, 2) hypothesis generation that motivates more focused and specific in vitro and in vivo testing in a more efficient manner, 3) expanding a systems biology understanding of plant bio-molecular systems by integrating multiple molecular pathway systems, and 4) exploring and testing the potential effects of exogenous inputs on the system of interest.

Relative to item (4), specifically, based on the current research herein, one of the more relevant and practical applications of this integrative in silico model of oxidative stress and C1 metabolism is to investigate how exogenous mechanisms such as genetic modification (GM), which have been shown to amplify oxidative stress in plants [4], may impact formaldehyde homeostasis and glutathione consumption in plants. Such future research may provide a new and alternative framework in the ongoing debate to understand whether genetically modified organisms (GMOs) are "substantially equivalent" to their non-GM counterparts.

\section{References}

[1] Demidchic, V. (2015) Mechanisms of Oxidative Stress in Plants: From Classical Chemistry to Cell Biology. Environmental and Experimental Botany, 105, 212-228. http://dx.doi.org/10.1146/annurev.arplant.50.1.601

[2] Foyer, C.H. and Shigeoka, S. (2011) Understanding Oxidative Stress and Antioxidant Functions to Enhance Photosynthesis. Plant Physiology, 155, 93-100. http://dx.doi.org/10.1021/bi961235j

[3] Arruda, S.C., Barbosa, H.S., Azevedo, R.A. and Arruda, M.A. (2013) Comparative Studies Focusing on Transgenic Through cp4EPSPS Gene and Non-Transgenic Soybean Plants: An Analysis of Protein Species and Enzymes. Journal of Proteomics, 93, 107-116. http://dx.doi.org/10.1104/pp.126.1.445

[4] Barbosa, H.S., Arruda, S.C., Azevedo, R.A. and Arruda, M.A. (2012) New Insights on Proteomics of Transgenic Soybean Seeds: Evaluation of Differential Expressions of Enzymes and Proteins. Analytical and Bioanalytical Chemistry, 402. 299-314. http://dx.doi.org/10.1104/pp.91.3.812

[5] Chew, O., Whelan, J. and Millar, A.H. (2003) Molecular Definition of the Ascorbate-Glutathione Cycle in Arabidopsis mitochondria Reveals Dual Targeting of Antioxidant Defenses in Plants. Journal of Biological Chemistry, 278, 4686946877. http://dx.doi.org/10.1016/S0891-5849(96)00185-2

[6] Ayyadurai, V.A. and Dewey, C.F. (2011) CytoSolve: A Scalable Computational Method for Dynamic Integration of Multiple Molecular Pathway Models. Cellular and Molecular Bioengineering, 4, 28-45.

http://dx.doi.org/10.1007/s12195-010-0143-x 
[7] Deonikar, P., Kothandaram, S., Mohan, M., Kollin, C., Konecky, P., Olovyanniko, R., Zamore, Z., Carey, B. and Ayyadurai1, V.A.S. (2015) Discovery of Key Molecular Pathways of C1 Metabolism and Formaldehyde Detoxification in Maize through a Systematic Bioinformatics Literature Review. Agricultural Sciences, 6, 571-585. http://dx.doi.org/10.4236/as.2015.65056

[8] Kothandaram, S., Deonikar, P., Mohan, M., Venugopal, V. and Ayyadurai, V.A.S. (2015) In Silico Modeling of C1 Metabolism. American Journal of Plant Sciences. [In Press] http://dx.doi.org/10.1016/B978-0-12-681903-8.50011-4

[9] Babbs, C.F. and Steiner, M.G. (1990) Simulation of Free Radical Reactions in Biology and Medicine: A New TwoCompartment Kinetic Model of Intracellular Lipid Peroxidation. Free Radical Biology and Medicine, 8, 471-485. http://dx.doi.org/10.1016/0891-5849(90)90060-V

[10] Polle, A. (2001) Dissecting the Superoxide Dismutase-Ascorbate-Glutathione-Pathway in Chloroplasts by Metabolic Modeling. Computer Simulations as a Step towards Flux Analysis. Plant Physiology, 126, 445-462. http://dx.doi.org/10.1104/pp.126.1.445

[11] Henle, E.S., Luo, Y. and Linn, S. (1996) $\mathrm{Fe}^{2+}, \mathrm{Fe}^{3+}$, and Oxygen React with DNA-Derived Radicals Formed during Iron-Mediated Fenton Reactions. Biochemistry, 35, 12212-12219. http://dx.doi.org/10.1021/bi961235j

[12] Mano, J. (2012) Reactive Carbonyl Species: Their Production from Lipid Peroxides, Action in Environmental Stress, and the Detoxification Mechanism. Plant Physiology and Biochemistry, 59, 90-97. http://dx.doi.org/10.1016/j.plaphy.2012.03.010

[13] Yokota, A., Kitaoka, S., Miura, K. and Wadano, A. (1985) Reactivity of Glyoxylate with Hydrogen Perioxide and Simulation of the Glycolate Pathway of C3 Plants and Euglena. Planta, 165, 59-67. http://dx.doi.org/10.1007/BF00392212

[14] Buettner, G.R., Ng, C.F., Wang, M., Rodgers, V.G. and Schafer, F.Q. (2006) A New Paradigm: Manganese Superoxide Dismutase Influences the Production of $\mathrm{H}_{2} \mathrm{O}_{2}$ in Cells and Thereby Their Biological State. Free Radical Biology and Medicine, 41, 1338-1350. http://dx.doi.org/10.1016/j.freeradbiomed.2006.07.015

[15] Wlodek, L. (1988) The Reaction of Sulfhydryl Groups with Carbonyl Compounds. Acta Biochimica Polonica, 35, 307317.

[16] Havir, E.A. and McHale, N.A. (1989) Enhanced-Peroxidatic Activity in Specific Catalase Isozymes of Tobacco, Barley, and Maize. Plant Physiology, 91, 812-815. http://dx.doi.org/10.1104/pp.91.3.812

[17] Achkor, H., et al. (2003) Enhanced Formaldehyde Detoxification by Overexpression of Glutathione-Dependent Formaldehyde Dehydrogenase from Arabidopsis. Plant Physiology, 132, 2248-2255. http://dx.doi.org/10.1104/pp.103.022277

[18] Goyer, A., et al. (2004) Characterization and Metabolic Function of a Peroxisomal Sarcosine and Pipecolate Oxidase from Arabidopsis. Journal of Biological Chemistry, 279, 16947-16953. http://dx.doi.org/10.1074/jbc.M400071200 


\section{Supplementary Materials}

Table S1. List of parameters used in in silico models of oxidative stress.

\begin{tabular}{|c|c|c|}
\hline $\begin{array}{l}\text { Kinetic } \\
\text { Parameter }\end{array}$ & Description & Reference \\
\hline $\mathrm{kO}_{2}^{-}$ & Rate constant for superoxide production & [S1] \\
\hline $\mathrm{kmO}_{2}^{-}$ & Michaelis Menten constant for superoxide production & [S1] \\
\hline $\mathrm{kFe}^{3}$ & Rate constant for the conversion of superoxide to oxygen with simultaneous reduction of $\mathrm{Fe}^{3+}$ to $\mathrm{Fe}^{2+}$ & [S2] \\
\hline $\mathrm{kH}_{2} \mathrm{O}_{2}$ & $\begin{array}{l}\text { Rate constant for the production of hydrogen peroxide } \\
\text { and oxygen from superoxide and } \mathrm{H}^{+} \text {(non-enzymatic) }\end{array}$ & [S3] \\
\hline kSOD & Rate constant for superoxide dismutase producing hydrogen peroxide from superoxide & [S3] \\
\hline $\mathrm{KmH}_{2} \mathrm{O}_{2}$ & Michaelis Menten constant for catalase induced conversion of $\mathrm{H}_{2} \mathrm{O}_{2}$ to $\mathrm{H}_{2} \mathrm{O}$ & [S4] \\
\hline kcata & Rate constant for catalase induced conversion of $\mathrm{H}_{2} \mathrm{O}_{2}$ to $\mathrm{H} 2 \mathrm{O}$ & [S4] \\
\hline $\mathrm{kFe}^{1}$ & $\begin{array}{l}\text { Fenton reaction rate constant (hydrogen peroxide forming hydroxyl } \\
\text { radical and anion with simultaneous conversion of } \mathrm{Fe}^{2+} \text { to } \mathrm{Fe}^{3+} \text { ) }\end{array}$ & [S2] \\
\hline kinitLR & Rate constant for lipid peroxidation reaction by hydroxyl radicals, forming lipid radicals & [S5] \\
\hline kLPO & Rate constant for the oxidation of lipid radicals & [S5] \\
\hline kLR1 & Rate constant for the formation of $\mathrm{L}^{*}$ and $\mathrm{LOOH}$ from $\mathrm{LH}$ and $\mathrm{LOO}^{*}$ & [S5] \\
\hline $\mathrm{kLRFe}^{1}$ & Rate constant for $\mathrm{Fe}^{2+}$ induced formation of $\mathrm{LO}^{*}$ from $\mathrm{LOOH}$ & [S6] \\
\hline $\mathrm{kLRFe}^{2}$ & Rate constant for $\mathrm{Fe}^{3+}$ induced formation of $\mathrm{LOO}^{*}$ from $\mathrm{LOOH}$ & [S6] \\
\hline kfrLOO & Rate constant for $\mathrm{LOO}^{*}$ fragmentation to alkane radical and aldehyde product & [S7] \\
\hline $\mathrm{kFe}^{4}$ & Rate constant for $\mathrm{OH}^{*}$ induced formation of $\mathrm{HO}_{2}{ }^{*}$ from $\mathrm{H}_{2} \mathrm{O}_{2}$ & [S2] \\
\hline $\mathrm{kFe}^{5}$ & Rate constant for $\mathrm{Fe}^{3+}$ induced formation of $\mathrm{HO}^{*}$ from $\mathrm{H}_{2} \mathrm{O}_{2}$ & [S2] \\
\hline $\mathrm{kFe}^{8}$ & Rate constant for $\mathrm{H}_{2} \mathrm{O}_{2}$ formation from $\mathrm{HO}_{2}{ }^{*}$ & {$[\mathrm{~S} 2]$} \\
\hline $\mathrm{kFe}^{9}$ & Rate constant for the conversion of $\mathrm{HO}_{2}{ }^{*}$ and $\mathrm{H}_{2} \mathrm{O}_{2}$ to $\mathrm{H}_{2} \mathrm{O}$ and $\mathrm{OH}^{*}$ & {$[\mathrm{~S} 2]$} \\
\hline $\mathrm{kFe}^{6}$ & Rate constant of Fe2+induced conversion of $\mathrm{OH}^{*}$ to $\mathrm{OH}^{-}$ & [S2] \\
\hline $\mathrm{kFe}^{7}$ & Rate constant for the conversion of $\mathrm{OH}^{*}$ and $\mathrm{HO}_{2}{ }^{*}$ to $\mathrm{H}_{2} \mathrm{O}$ and $\mathrm{O}_{2}$ & [S2] \\
\hline $\mathrm{kdH}_{2} \mathrm{O}$ & Dissociation rate of $\mathrm{H}_{2} \mathrm{O}$ to $\mathrm{H}^{+}$and $\mathrm{OH}-$ & [S8] \\
\hline $\mathrm{KH}_{2} \mathrm{O}$ & Association rate of $\mathrm{H}^{+}$and $\mathrm{OH}$ - to $\mathrm{H}_{2} \mathrm{O}$ & [S8] \\
\hline kAPX & Rate constant for APX induced conversion of Ascorbate to MDA & [S3] \\
\hline KAPX & Michaelis Menten constant for APX induced conversion of ASC to MDA & {$[\mathrm{S} 3]$} \\
\hline КАРХH & Michaelis Menten constant for APX induced conversion of $\mathrm{H}_{2} \mathrm{O}_{2}$ to $\mathrm{H}_{2} \mathrm{O}$ & [S3] \\
\hline $\mathrm{k} \_\mathrm{ASCH} \mathrm{O}_{2} \mathrm{O}_{2}$ & Rate constant for ASC and $\mathrm{H}_{2} \mathrm{O}_{2}$ & [S3] \\
\hline k_ASCO ${ }_{2}$ & Rate constant for superoxide reacting with ascorbate & [S3] \\
\hline kMDAR & Rate constant for molecular MDAR activity & [S3] \\
\hline KMDARM & Michaelis Menten constant of MDAR for MDA & {$[\mathrm{S} 3]$} \\
\hline KMDARN & Michaelis Menten constant of MDAR for NADPH & [S3] \\
\hline k_MDAMDA & Apparent rate constant of MDA & [S3] \\
\hline kDAR & Rate constant for molecular DAR activity & [S3] \\
\hline KDAR & Michaelis Menten constant of DAR for DHA & [S3] \\
\hline KDARG & Michaelis Menten constant of DAR for GSH & [S3] \\
\hline k_DHAGSH & Apparent rate constant of GSH and DHA & [S3] \\
\hline kGPxr & Rate constant of reduced GPx with $\mathrm{H}_{2} \mathrm{O}_{2}$ & [S9] \\
\hline kGPxo & Rate constant of oxidized GPx with GSH to form intermediate GSGPx & [S9] \\
\hline kGSSG & Rate constant of GSGPx with GSH to recycle reduced Gpx & [S9] \\
\hline kGR & Rate constant for molecular GR activity & [S3] \\
\hline KGR & Michaelis Menten constant of GR for GSSG & [S3] \\
\hline KGRN & Michaelis Menten constant of GR for NADPH & [S3] \\
\hline kNAP & Rate constant for the conversion of NADP to NADPH & [S3] \\
\hline
\end{tabular}




\section{Appendix A: List of Keywords}

1. Oxidative stress in plants

2. Effect of oxidative stress on $\mathrm{C} 1$ metabolism

3. Oxidative stress signaling pathways-pdf

4. Hydrogen peroxide and glutathione

5. Hydrogen peroxide and glutathione peroxidase in plants

6. Superoxide production AND photosynthesis

7. Superoxide AND lipid peroxidation

8. Perhydroxyl radical AND oxidative stress in plants

9. Perhydroxyl radical AND oxidative stress in plants-pdf

10. Fenton reaction AND oxidative stress in plants

11. Ascorbate glutathione cycle and photosystem reactions

12. Ascorbate glutathione cycle localization in chloroplast

13. Factors affecting formaldehyde dehydrogenase activity AND oxidative stress

14. Formaldehyde dehydrogenase acting on lipid peroxide

15. Lipid peroxide as substrate for formaldehyde dehydrogenase

16. Kinetics of iron uptake in maize

17. Catalase AND oxidative stress

18. Mathematical model of ascorbate glutathione cycle

19. ROS upregulates catalase expression in plants

20. Competitive inhibitors of formaldehyde dehydrogenase AND plant

21. Requirement of GSH for formaldehyde dehydrogenase activity

22. Glutathione depletion and formaldehyde dehydrogenase

23. Hydrogen peroxide levels AND ascorbate glutathione cycle

24. Iron AND oxidative stress AND glutathione level AND plants

\section{Supplementary Materials References}

[S1] Asada, K. (1999) The Water-Water Cycle in Chloroplasts: Scavenging of Active Oxygens and Dissipation of Excess Photons. Annual Review of Plant Physiology and Plant Molecular Biology, 50, 601-639. http://dx.doi.org/10.1146/annurev.arplant.50.1.601

[S2] Henle, E.S., Luo, Y. and Linn, S. (1996) $\mathrm{Fe}^{2+}, \mathrm{Fe}^{3+}$, and Oxygen React with DNA-Derived Radicals Formed during Iron-Mediated Fenton Reactions. Biochemistry, 35, 12212-12219. http://dx.doi.org/10.1021/bi961235j

[S3] Polle, A. (2001) Dissecting the Superoxide Dismutase-Ascorbate-Glutathione-Pathway in Chloroplasts by Metabolic Modeling. Computer Simulations as a Step towards Flux Analysis. Plant Physiology, 126, 445-462. http://dx.doi.org/10.1104/pp.126.1.445

[S4] Havir, E. and McHale, N. (1989) Enhanced-Peroxidatic Activity in Specific Catalase Isozymes of Tobacco, Barley, and Maize. Plant Physiology, 91, 812-815. http://dx.doi.org/10.1104/pp.91.3.812

[S5] Antunes, F., Salvador, A., Marinho, H.S., Alves, R. and Pinto, R.E. (1996) Lipid Peroxidation in Mitochondrial Inner Membranes. I. An Integrative Kinetic Model. Free Radical Biology and Medicine, 21, 917-943. http://dx.doi.org/10.1016/S0891-5849(96)00185-2

[S6] Xue, C., Chou, C.-S., Kao, C.-Y., Sen, C.K. and Friedman, A. (2013) Propagation of Cutaneous Thermal Injury: A Mathematical Model. Wound Repair and Regeneration, 20, 114-122. http://dx.doi.org/10.1111/j.1524-475X.2011.00759.x

[S7] Pratt, D.A., Tallman, K.A. and Porter, N.A. (2011) Free Radical Oxidation of Polyunsaturated Lipids: New Mechanistic Insights and the Development of Peroxyl Radical Clocks. Accounts of Chemical Research, 44, 458-467. http://dx.doi.org/10.1021/ar200024c

[S8] Stillinger, F.H. (1978) Proton Transfer Reactions and Kinetics in Water. Theoretical Chemistry, 3, 177-234. http://dx.doi.org/10.1016/b978-0-12-681903-8.50011-4

[S9] Buettner, G.R., Ng, C.F., Wang, M., Rodgers, V.G.J. and Schafer, F.Q. (2006) A New Paradigm: Manganese Superoxide Dismutase Influences the Production of $\mathrm{H}_{2} \mathrm{O}_{2}$ in Cells and Thereby Their Biological State. Free Radical Biology and Medicine, 41, 1338-1350. http://dx.doi.org/10.1016/j.freeradbiomed.2006.07.015 